\title{
REGISTRO DE POLIPLOIDIA EN LA ALMEJA DULCEACUICOLA MUSCULIUM ARGENTINUM (D'ORBIGNY 1835) (SPHAERIIDAE, VENEROIDA)
}

\author{
RECORD OF POLYPLOIDY IN THE FRESHWATER CLAM MUSCULIUM \\ ARGENTINUM (D'ORBIGNY 1835) (SPHAERIIDAE, VENEROIDA)
}

\author{
Pedro Jara-Seguel, Santiago Peredo \& Esperanza Parada \\ Escuela de Ciencias Biológicas y Químicas, Facultad de Recursos Naturales, Universidad Católica de Temuco, Casilla \\ 15-D, Temuco-Chile. Email: pjara@uct.cl
}

\section{RESUMEN}

La poliploidía es un carácter inusual en moluscos bivalvos. Sin embargo, datos citogenéticos documentados para especies de la subfamilia Sphaeriinae (familia Sphaeriidae) del hemisferio norte muestran números cromosómicos que varían desde 36 hasta ca. 247. La almeja dulceacuícola Musculium argentinum (D’Orbigny 1835) presenta un número cromosómico $2 \mathrm{n}=c a$. 130, lo cual muestra que la poliploidía también estaría presente en esféridos sudamericanos. Estos antecedentes citogenéticos sugieren que Sphaeriinae podría constituir un complejo poliploide maduro.

Palabras claves: Sphaeriidae, Musculium argentinum, poliploidía, complejo poliploide.

\section{ABSTRACT}

The polyploidy is an unusual character in bivalve mollusks. However, cytogenetic data documented for north-hemispheric species of the subfamily Sphaeriinae (family Sphaeriidae), showed chromosome numbers ranging from 36 to $c a$. 247. The freshwater clam Musculium argentinum (D’Orbigny 1835) has a polyploid chromosome number $2 \mathrm{n}=c a$. 130 , which shows that polyploidy likewise may be present in South American sphaeriids. These cytogenetic data suggest that the subfamily Sphaeriinae could constitute a mature polyploid complex.

Keywords: Sphaeriidae, Musculium argentinum, polyploidy, polyploid complex.

\section{INTRODUCCION}

La poliploidía es considerada un carácter inusual en moluscos bivalvos (Nakamura 1985). Sin embargo, evidencia citogenética recopilada por Lee (1999) indica que las especies de los géneros dulceacuícolas Sphaerium Scopoli, Musculium Link y Pisidium Pfeiffer, adscritos a la subfamilia Sphaeriinae (Sphaeriidae), serían poliploides. A la fecha, se han documentado antecedentes citogenéticos para 10 especies de Sphaeriinae que habitan en el hemisferio norte, las cuales se caracterizan por presentar los más altos niveles de ploidía descritos dentro de la clase bivalvia, con valores desde $2 n$ hasta $13 n$ y con números cromosómicos que varían desde 36 hasta ca. 247 (Lee 1999). Lee \& Ó Foighil (2002), en base a evidencia molecular, sugieren que esta pronunciada poliploidización sería una característica ancestral para el linaje Sphaerium-Musculium y constituiría un carácter que podría ser predominante para especies de los géneros cosmopolitas Sphaerium, Musculium y Pisidium. Esta predominancia de la poliploidía en Sphaeriinae fue previamente discutida por Lee (1999), quien señala, 
además, que la realización de estudios citogenéticos en otras especies de esféridos permitiría determinar si existe poliploidía en más taxones de esta subfamilia. En Chile, la familia Sphaeriidae está representada por los géneros Sphaerium (2 especies), Musculium (2 especies) y Pisidium (7 especies), cuyas especies se distribuyen en la zona norte, central y sur, en cuerpos de agua localizados desde el nivel del mar hasta los 3.800 m.s.n.m. en la zona Andina (Parada \& Peredo 2002). Sobarzo et al. (2002) registraron por primera vez la presencia de Musculium argentinum(D'Orbigny 1835) en aguas continentales chilenas, especie que previamente fue redescrita por Ituarte (1996) para Argentina. Con este antecedente taxonómico, aumentó a 11 el número de especies de esféridos para la malacofauna dulceacuícola de Chile, constituyendo, por lo tanto, el grupo de bivalvos de mayor diversidad en aguas continentales del país. Sin embargo, a pesar de esta alta diversidad, antecedentes biológicos (i. e., genéticos, reproductivos, ecológicos) para especies chilenas de esta subfamilia, así como para otras especies del hemisferio sur, aún no han sido documentados.

Si, como proponen Lee (1999) y Lee \& Ò Foighil (2002), la poliploidía es un carácter ancestral y predominante dentro de Sphaeriinae, entonces podemos predecir que $M$. argentinumes una especie poliploide. Con el objeto de someter a prueba esta hipótesis, en este trabajo se describe el número cromosómico de Musculium argentinum, antecedente que se complementa con la información citogenética existente para esféridos del hemisferio norte.

\section{MATERIAL Y METODOS}

Se colectaron individuos adultos de Musculium argentinum en la población tipo registrada por Sobarzo et al. (2002), localizada en el canal de desagüe de la Piscicultura de Lautaro, derivado del Río Cautín (38³2'S; $72^{\circ} 27^{\prime} \mathrm{W}$ ), Chile. Ejemplares de esta población se encuentran depositados en la colección zoológica de la Escuela de Ciencias Biológicas y Químicas, Facultad de Recursos Naturales, Universidad Católica de Temuco.

Juveniles de 500 a $1.000 \mu \mathrm{m}$ de longitud se extrajeron, mediante disección, desde la hemibranquia de individuos grávidos. Los juveniles fueron tratados con colchicina $0.05 \%$ durante $2 \mathrm{~h}$, hipotonizados en una mezcla de agua destilada - agua de río (1:1 v/v) por $45 \mathrm{~min}$, fijados en etanol - ácido acético $(3: 1 \mathrm{v} / \mathrm{v})$ por $24 \mathrm{~h}$ y coloreados con la reacción de Feulgen. Metafases mitóticas fueron obtenidas mediante la técnica de aplastado de juveniles y las preparaciones se montaron en Entellan (Merck). Las metafases fueron fotografiadas con una cámara digital Olympus C-5050 conectada a un microscopio OLympus CX31 y las imágenes se almacenaron en formato TIFF de alta resolución. Se seleccionaron 15 imágenes de metafases, de las cuales se obtuvieron impresiones fotográficas ampliadas. En las fotomicrografías se realizó el conteo de cromosomas y se determinó la longitud de los cromosomas más grandes del complemento.

\section{RESULTADOS Y DISCUSION}

Las células somáticas de los juveniles analizados en M. argentinum presentan ca. 130 cromosomas (rango: 100 - 130, valor modal: 130) (Fig. 1). Este número de cromosomas es menor al documentado por Burch et al. (1998) para M. securis (Prime 1851) $(2 \mathrm{n}=$ ca. 247) y está dentro del rango de poliploidía descrito para especies de Sphaeriinae del hemisferio norte (ca. 68 - 247 cromosomas) (Woods 1931, Barsiené et al. 1996, Burch et al. 1998, Lee 1999 y Lee \& Ó Foighil 2002). Por otra parte, Sphaerium corneum (Linnaeus 1758$)(2 \mathrm{n}=36)$ es, a la fecha, la única especie para la cual se ha descrito un número cromosómico diploide dentro de Sphaeriinae (Keyl 1956) (Tabla I).

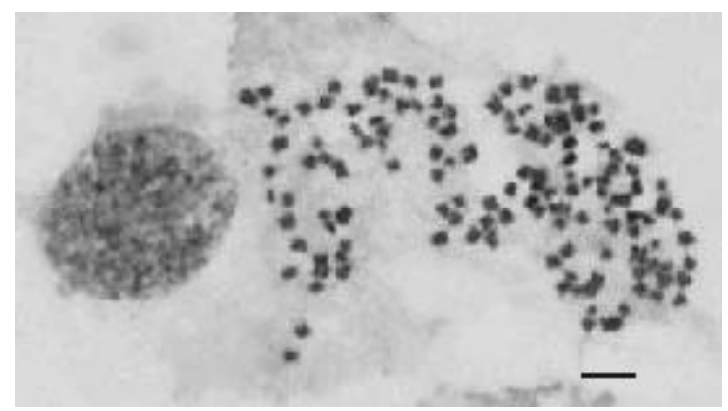

Figura 1. Cromosomas mitóticos $(2 \mathrm{n}=c a$. 130) de Musculium argentinum. Barra $=5 \mu \mathrm{m}$.

Figure 1. Mitotic chromosomes $(2 \mathrm{n}=c a$. 130) of Musculium argentinum. Bar $=5 \mu \mathrm{m}$. 
Gayana 69(1), 2005

TABLA I. Número cromosómico y localidades de colecta de especies de Sphaeriinae analizadas a la fecha. (Modificada de Lee 1999).

TABLE I. Chromosome number and collection locality of species of Sphaeriinae analysed so far. (Modified from Lee 1999).

\begin{tabular}{llll}
\hline & Número & $\begin{array}{l}\text { Localidad } \\
\text { de colecta }\end{array}$ & \\
Especie & cromosómico & & Referencias \\
\hline Sphaerium striatinum & $c a .68,98$ & Michigan, USA & Woods (1931) \\
& $c a .152$ & Michigan, USA & Lee (1999) \\
S. corneum & 36 & Europa & Keyl (1956) \\
S. occidentale & $c a .209$ & Michigan, USA & Burch et al. (1998) \\
S. simile & $>100$ & Michigan, USA & Lee \& Ó Foighil (2002) \\
Pisidium casertanum & $c a .150,180$ & Spanish Mountain & Barsiene et al. $(1996)$ \\
& $c a .190$ & Spanish Mountain & Burch et al. (1998) \\
P. compressum & $>100$ & Michigan, USA & Lee \& Ó Foighil (2002) \\
P. adamsi & $>100$ & Michigan, USA & Lee \& Ó Foighil (2002) \\
P. dubium & $>200$ & Michigan, USA & Lee \& Ó Foighil (2002) \\
P. coreanum & $c a .190$ & Korea & Park et al. (2002) \\
Musculium securis & $c a .247$ & Michigan, USA & Burch et al. (1998) \\
M. argentinum & $c a .130$ & Río Cautín, Chile & Presente estudio \\
\hline
\end{tabular}

Entre los caracteres más importantes observados en el complemento cromosómico de estos bivalvos dulceacuícolas, además de la poliploidía, están el pequeño tamaño de los cromosomas y la alta variabilidad en el número cromosómico a nivel interespecífico. En M. argentinum, el tamaño absoluto de los cromosomas más grandes es de $1.9 \mu \mathrm{m}$, valor que es menor al descrito por Lee (1999) para Sphaerium striatinum (Lamarck 1818), especie en la que los cromosomas de mayor longitud miden, aproximadamente, $5.0 \mu \mathrm{m}$. Respecto al número cromosómico, existe una alta variación al hacer comparaciones interespecíficas dentro de cada género, característica que es diferente a lo descrito tanto para bivalvos marinos (i.e., Mytilus, Ostrea, Crassostrea, Pecten) (Méndez et al. 2001), así como para bivalvos dulceacuícolas (i. e., Unio) (Barsiené 1994), en los cuales se observa constancia en el número de cromosomas entre las especies de cada género.

Los resultados del presente trabajo, basados principalmente en el número cromosómico, respaldan lo señalado por Lee (1999) y Lee \& Ó Foighil (2002) respecto de que la poliploidía sería un carácter predominante dentro de Sphaeriinae, por lo que es altamente probable que las otras 10 especies chilenas, adscritas a Sphaerium, Musculium y Pisidium, sean también poliploides. El origen de esta poliploidía descrita en Sphaeriinae, así como su ventaja adaptativa para las poblaciones de estas especies, aún no es conocido. Otto \& Whitton (2000) señalan que la hibridación interespecífica y la subsecuente duplicación del genoma es la vía más eficiente y repentina para realizar doblamiento genómico en eucariotas. En base a estos argumentos, Lee (2001) sugiere un origen por hibridación interespecífica para las especies poliploides de Sphaeriinae, las cuales, a través de sucesivos procesos de amplificación de sus genomas, habrían alcanzado los altos niveles de ploidía descritos, siendo la alopoliploidía el mecanismo de evolución más probable. Esta afirmación ha sido respaldada por Lee \& Ó Foighil (2002), quienes secuenciaron alelos del gen para 6fosfogluconato deshidrogenasa en varias especies de esféridos. De acuerdo con esos datos moleculares, dichos autores sugieren que patrones ancestrales de poliploidización podrían subyacer a la presente diversidad de estos moluscos dulceacuícolas (aproximadamente 79 especies de Sphaeriinae), evidenciando para el linaje Sphaerium-Musculium, eventos sucesivos de duplicación genómica que 
podrían haberse iniciado en tiempos previos al Mioceno. Pisidium, en tanto, habría estado sujeto a eventos más recientes de poliploidización.

Aunque la poliploidía ha sido señalada como inusual en bivalvos, fue descrita también en especies de los géneros marinos Lasaea $(4 \mathrm{n}=94$-104) (Lasaeidae) (Thiriot-Quiévreux et al. 1988, Ó Foighil \& ThiriotQuiévreux 1999) y Mytilus (M. trossulus Gould 1850, 2n = 86) (Mytilidae) (González-Tizónet al.2000). Igualmente, algunas poblaciones de las especies dulceacuícolas Corbicula fluminea (Müller 1774) $(3 n=54$ y $4 n=72)$ y C. leana $($ Prime 1864$)(3 n=54)$ (Corbiculidae) (Qiu et al. 2001) son también poliploides. Este descubrimiento de poliploidía en bivalvos, en conjunto con los datos previos existentes para gastrópodos (Burch 1964, Burch \& Huber 1966), ha enfatizado el rol evolutivo que tendría la amplificación genómica en los moluscos, dando paso también, a nuevas líneas de trabajo orientadas a determinar los probables mecanismos reproductivos que podrían estar asociados a este proceso de poliploidización (i.e., androgénesis, ginogénesis), según se desprende de los antecedentes documentados por Woods (1931), Keyl (1956), Komaru et al. (1997), Komaru et al. (1998), Ó Foighil \& Thiriót-Quevreux (1999) y Lee (1999).

Finalmente, al considerar la amplia distribución geográfica que presentan las especies de la subfamilia Sphaeriinae (en los hemisferios norte y sur), con una notable predominancia de los taxa poliploides (10 especies) por sobre los diploides (1 especie) y en conjunto con aquellos patrones ancestrales de amplificación genómica discutidos por Lee \& Ó Foighil (2002), sugerimos que esta subfamilia reuniría las condiciones para ser considerada un complejo poliploide maduro de acuerdo a la clasificación de Stebbins (1971). Este aspecto evolutivo aún no ha sido discutido para este grupo de bivalvos, por lo que la realización de estudios citogenéticos y moleculares, que incluyan un mayor número de especies, tanto del hemisferio norte como del hemisferio sur, permitirían recopilar antecedentes para respaldar o rechazar esta hipótesis.

\section{AGRADECIMIENTOS}

Los autores agradecen a la Dirección General de Investigación de la Universidad Católica de Temuco por el financiamiento a través de los proyectos DGIUCT $\mathrm{N}^{\circ}$ 2005-4-02 y N $\mathrm{N}^{\circ}$ 2005-4-03. A Daniel Rozas, por su colaboración en las actividades de colecta.

\section{BIBLIOGRAFIA}

BARSIENÉ, J. 1994. Chromosome set changes in mollusks from highly polluted habits pp: 434 - 447 in A. R. Beaumont (ed.), Genetics and Evolution of Aquatic Organisms. Chapman \& Hall: London.

Barsiené, J., G. T APIA \& D. Barsyte. 1996. Chromosome of molluscs inhabiting some mountain spring of eastern Spain. Journal of Molluscan Studies 62: 539-543.

Burch, J. 1964. Cytological studies of Planorbidae (Gastropoda: Basommatophora). I. The African subgenus Bulinus s.s. Malacologia 1: 387-400.

Burch, J. \& J. Huber. 1966. Polyployd in mollusks. Malacologia 5: 41-43.

Burch, J., G. Park \& E., Chung. 1998. Michigan‘s polyploid clams. Michigan Academician 30: 351-352.

González-Tizón, A., A. Martínez-Lage, J. Ausio \& J. MÉndez. 2000. Polyploidy in a natural population of mussel, Mytilus trossulus. Genome 43: 409-411.

Ituarte C. 1996. Argentine Species of Pisidium Pfeiffer, 1821, and Musculium Link, 1807 (Bivalvia: Sphaeriidae). The Veliger 39 (3): 189-203.

KeYL, H. 1956. Beobachtungen über die $\sigma^{\star}$-meiose der muschel Sphaerium corneum. Chromosoma 8: 1217.

Komaru, A., K. Konishi, I. Nakayama, T. Koboyashi, H. Sakai \& K. Kawamura. 1997. Hermaphroditic freshwater clams in the genus Corbicula produce non-reductional spermatozoa with somatic DNA content. Biological Bulletin 193: 320-323.

Komaru, A., T. Kawagishi \& K. Konishi. 1998. Cytological evidence of spontaneous androgenesis in the freshwater clam Corbicula leana Prime. Dev. Genes Evol. 208: 46-50.

LEE, T. 1999. Polyploidy and meiosis in the freshwater clam Sphaerium striatinum (Lamarck) and chromosome numbers in the Sphaeriidae (Bivalvia, Veneroida). Cytologia 64: 247-252.

LeE, T. 2001. Systematic revision of the Sphaeriinae (Mollusca, Bivalvia, Veneroida, Sphaeriidae). D. Phil Thesis, University of Michigan. $210 \mathrm{pp}$.

Lee, T. \& D. Ó Foighil. 2002. 6-Phosphogluconate deshydrogenase (PGD) allele phylogeny is incongruent with a recent origin of polyploidization in some North American Sphaeriidae (Mollusca, Bivalvia). Molecular Phylogenetics and Evolution 25: 112-124.

Méndez, J., A. Insua \& J. López-Piñón. 2001. Caracterización citogenética en moluscos bivalvos, pp: 15-48. En: Méndez-Felpeto (Ed.), Los moluscos bivalvos: Aspectos citogenéticos, 
moleculares y aplicados. Universidad de la Coruña, Xaneiro. $240 \mathrm{pp}$.

NAKAMURA, H. 1985. A review of molluscan cytogenetic information based on the CISMOCH-Computerized Index System for Molluscan Chromosomes. Bivalvia, Polyplacophora and Cephalopoda. Venus 44: 193-225.

Ó Foighil D. \& K. T hiriot-Quiévreux. 1999. Sympatric Australian Lasea species (Mollusca: Bivalvia) differ in their ploidy levels, reproductive modes and developmental models. Zoological Journal of the Linnean Society 127: 477-494.

Oтto, S. \& J. Whitton. 2000. Polyploidy incidence and evolution. Annual Review of Genetics 34: 401437.

Parada, E. \& S. Peredo. 2002. Estado actual de la taxonomía de bivalvos dulceacuícolas chilenos: progresos y conflictos. Revista Chilena de Historia Natural 75: 691-701.

PARK, G., T. Yong \& J. Burch. 2002. Chromosomes of Pisidium coreanum (Bivalvia: Veneroida:
Corbiculoidea, Pisidiidae), a Korean freshwater clam. Malacologia 44: 165-168.

Qiu, A., A. Shi \& A. Komaru. 2001. Yellow and brown color morphs of Corbicula fluminea (Bivalvia: Curbiculidae) from Sichuan Province, China, are triploids and tetraploids. Journal of Shellfish Research 20 (1): 323- 328.

Sobarzo, C., P. Jara-Seguel, S. Peredo \& E. Parada. 2002. Primer registro de Musculium argentinum (D‘orbigny 1835) (Bivalvia: Sphaeriidae) en aguas continentales chilenas. Gayana 66 (1): 3943.

StebBins, G. 1971. Chromosomal evolution in higher plants. Edward Arnold Publishing, London. 215 pp

Thiriot-Quiévreux, K., J. Soyer, F. De Bovée \& P. Albert. 1988. Unusual chromosome complement in the brooding bivalve Lasaea consanguinea. Genetica 76: $143-151$.

Woods, F. 1931. History of the germ cells in Sphaerium striatinum (Lam.). Journal of Morphology 51: 545-595. 